\title{
PI3K/mTOR Inhibitors in the Treatment of Luminal Breast Cancer. Why, When and to Whom?
}

\author{
Francesco Schettini $^{a}$ Giuseppe Buono ${ }^{a}$ Meghana V. Trivedi ${ }^{b, c}$ Sabino De Placido ${ }^{a}$ \\ Grazia Arpino $^{\text {a }}$ Mario Giuliano ${ }^{\mathrm{a}, \mathrm{b}}$ \\ ${ }^{a}$ Department of Clinical Medicine and Surgery, University Federico II, Naples, Italy; \\ ${ }^{b}$ Lester and Sue Smith Breast Center, Baylor College of Medicine, Houston, TX, USA; \\ ${ }^{c}$ Department of Clinical Sciences and Administration, University of Houston, College of Pharmacy, Houston, TX, USA
}

\section{Keywords}

Breast cancer - Endocrine therapy - Targets - PI3K . mTOR

\section{Summary}

Estrogen receptor (ER) signaling represents the main driver of tumor growth and survival in luminal breast cancer (BC). Despite the efficacy of endocrine agents, many patients with luminal $B C$ do not respond to endocrine therapy and many others develop endocrine resistance over time, due to the activation of escape pathways such as the PI3K/AKT/mTOR signaling. Several clinical trials have demonstrated the efficacy of mTOR and PI3K inhibitors in overcoming endocrine resistance in hormone receptor-positive human epidermal growth factor receptor 2 (HER2)-negative metastatic $\mathrm{BC}$ (MBC) patients. Nevertheless, to date, everolimus is the only agent targeting the $\mathrm{PI} 3 \mathrm{~K} / \mathrm{mTOR}$ pathway that has been approved for clinical use. Recently, the introduction of CDK 4/6 inhibitors into clinical practice has significantly changed the therapeutic scenarios in luminal MBC. In the absence of direct comparisons among the new treatment combinations and predictive biomarkers of response, the choice of optimal therapeutic algorithms is very challenging. Future trials should focus on identifying more effective and safe combination therapies and defining the best treatment sequences in luminal BC.

(c) 2017 S. Karger GmbH, Freiburg

Francesco Schettini and Giuseppe Buono contributed equally to this article.

\section{Introduction}

Luminal breast cancer (BC) accounts for approximately $60-70 \%$ of all breast tumors $[1,2]$ and is divided into 3 subtypes including luminal $\mathrm{A}$ and luminal $\mathrm{B}$, with or without amplification/overexpression of human epidermal growth factor receptor 2 (HER2) [1]. Tumor cell growth in HER2-negative luminal BC is primarily driven by estrogen receptor (ER) signaling [2]. Despite the proven efficacy of endocrine treatments, intrinsic (de novo) and acquired endocrine resistance occurs in a significant percentage of patients. Different molecular mechanisms have been proposed as the causes of endocrine resistance, including loss of ER expression, altered activity of ER co-regulators, deregulation of apoptosis and cell cycle, ER gene mutations, and hyperactive receptor tyrosine kinase (RTK) downstream pathways [2]. In this review, we point out the key role of the PI3K/AKT/mTOR pathway in the development of endocrine resistance. We also summarize clinical evidence supporting $\mathrm{PI} 3 \mathrm{~K} / \mathrm{mTOR}$ inhibition in hormone receptor (HR)-positive HER2-negative $\mathrm{BC}$, and debate the role of PI3K/mTOR inhibitors in the current therapeutic scenario.

\section{Role of PI3K/mTOR Pathway in Endocrine Resistance}

ER signaling mediates tumor growth and survival through different molecular mechanisms, involving nuclear/genomic and non-nuclear/non-genomic functions [3]. The first is activated when estrogens bind ER protein leading to the formation of a transcription machinery that exerts the so-called ER classical genomic activity, by directly binding specific promoter regions of target genes, known as ER elements (EREs) [3]. Of note, ER classical genomic activity is effectively inhibited by endocrine agents. In ad-

\section{KARGER}

(c) 2017 S. Karger GmbH, Freiburg

Fax +497614520714
Mario Giuliano. MD, PhD

Department of Clinical Medicine and Surgery

University Federico II

Naples, Italy

m.giuliano@unina.it 
dition, estrogen/ER complexes can also interact with other transcription factors, such as AP-1/SP-1 family members, modulating alternative transcriptional programs [3]. On the other hand, the non-nuclear ER mechanism of action is mediated by an intensive molecular crosstalk between ER and RTK pathways, in which estrogen/ER complexes, present in the cytoplasm or at cell membrane level, directly interact with RTKs and their downstream signaling pathways, including the Ras/MAPK and the PI3K/AKT/ mTOR pathways [3]. Importantly, an overactive PI3K/mTOR cascade can play a crucial role in the development of endocrine resistance as it can function as an escape pathway able to bypass the ER signaling blockade. Moreover, it can also modulate both alternative genomic and non-genomic ER activities, and ultimately sustain resistant cell growth [3].

$\mathrm{PI} 3 \mathrm{~K}$ is a heterodimeric tyrosine kinase formed by a catalytic subunit (p110 isoforms $\alpha, \beta$ or $\delta$ ) and a regulatory subunit (p85, p55 or p50). Activation of PI3K leads to the phosphorylation of several substrates, including membrane phosphatidylinositol (PIP). The conversion from PIP2 to PIP3 is responsible for the activation of AKT. This in turn phosphorylates several molecules, including TSC2 with consequent activation of the mTOR complex, and ER itself, leading to its activation in a ligand-independent manner [4]. The p110a subunit also interacts with the activated form of the proto-oncogene Ras, suggesting a crosstalk between the MAPK and PI3K pathways [5]. Finally, the serine/threonine kinase mTOR is the catalytic subunit of the mTORC1 and 2 complexes. mTORC1 mediates cell growth, as well as protein and lipid synthesis, whereas mTORC2 stimulates proliferation and cell survival [5]. Deregulation of this complex pathway can occur as a consequence of activating mutations in the PI3K catalytic subunit gene PIK3CA, loss of function of PI3K-negative regulators (PTEN, TSC, and LKB1), and/or overexpression of upstream RTKs, such as epidermal growth factor receptor (EGFR), HER2, and insulin-like growth factor receptor 1 (IGFR1) [4-7].

Several preclinical studies have clearly demonstrated the key role of PI3K/AKT/mTOR pathway in the development of acquired endocrine resistance in luminal $\mathrm{BC}$ and shown the capability of $\mathrm{PI} 3 \mathrm{~K} / \mathrm{mTOR}$ inhibitors in overcoming resistance $[6,7]$. This led to the initiation of vast clinical research programs testing mTOR and $\mathrm{PI} 3 \mathrm{~K}$ inhibition associated with endocrine therapy (ET) in luminal metastatic $\mathrm{BC}(\mathrm{MBC})$.

\section{Clinical Development of mTOR and PI3K Inhibitors in Luminal MBC}

The mTORC1 inhibitor everolimus was the first oral agent targeting the PI3K/AKT/mTOR pathway to be introduced into clinical practice in luminal $\mathrm{MBC}$, following the positive results of the BOLERO-2 trial [8]. In this phase III randomized study, the combination of everolimus and exemestane was compared with exemestane plus placebo in 724 postmenopausal $\mathrm{HR}^{+} / \mathrm{HER} 2^{-} \mathrm{MBC}$ patients who had disease recurrence or progression after previous therapy with a non-steroidal aromatase inhibitor (NSAI). The study showed a remarkable improvement in median progressionfree survival (PFS) for the experimental arm (6.9 vs. 2.8 months, according to local assessment; hazard ratio 0.43, p < 0.001) [8]. Nevertheless, no statistically significant difference in overall survival (OS) was found, despite a numerical advantage of the experimental arm (31.0 vs. 26.6 months; hazard ratio $0.89, \mathrm{p}=0.1426$ ) [9]. The combination therapy showed a worse safety profile, with the most common grade 3 or 4 adverse events being stomatitis ( $8 \%$ vs. $1 \%)$, hyperglycemia ( $4 \%$ vs. $<1 \%)$, anemia ( $6 \%$ vs. $<1 \%)$, fatigue ( $4 \%$ vs. $1 \%)$, dyspnea ( $4 \%$ vs. $1 \%$ ) and pneumonitis ( $3 \%$ vs. $0 \%$ ) [8]. These results confirmed the data previously reported in the phase II TAMRAD trial by the combination of everolimus plus tamoxifen [10].

Subsequently, the randomized phase III trial HORIZON failed to demonstrate any survival improvement in postmenopausal patients with luminal MBC treated with the combination of the mTOR inhibitor temsirolimus plus letrozole versus letrozole plus placebo, as first-line therapy in the absence of prior exposure to aromatase inhibitors (AIs) (PFS hazard ratio 0.90, $\mathrm{p}=0.25$ ) [11]. More recently the randomized PrECOG phase II trial provided evidence of efficacy for the combination of everolimus and fulvestrant in AI-resistant luminal MBC. In this study, which enrolled postmenopausal patients pre-treated with an $\mathrm{AI}$ in the adjuvant or metastatic disease setting, PFS was significantly improved by the combination arm compared with fulvestrant alone (10.4 vs. 5.1 months; hazard ratio $0.6, \mathrm{p}=0.02$ ), although no significant difference in OS was observed $(p=0.79)[12]$. The everolimus-fulvestrant arm was associated with higher toxicity than the control arm, in agreement with that observed in the BOLERO2 trial [12]. Another phase II single-arm trial, the BOLERO-4, tested the combination of everolimus + letrozole as first-line treatment in 202 postmenopausal patients with luminal MBC. After disease progression, patients could also receive everolimus + exemestane until further disease progression or unacceptable toxicity. The updated results, presented at the 2017 American Society of Clinical Oncology (ASCO) meeting, showed a median PFS of 21.7 months (95\% confidence interval (CI) 18.1-23.9), with an overall response rate (ORR) of $43.6 \%$ and a clinical benefit rate (CBR) of $74.3 \%$. 42 patients progressing on first-line treatment received everolimus + exemestane as a secondline therapy. In this smaller group, median PFS was 3.7 months (95\% CI 1.8-9.1), ORR was 4.8\% and CBR 21.4\%. OS analyses are still ongoing [13].

During the clinical development of mTOR inhibitors, translational studies have suggested that the efficacy of these agents might be limited by the activation of a feedback loop leading to upregulation of the PI3K/AKT signaling pathway, mediated by IGF1R signaling $[14,15]$. Basing on this evidence, 2 randomized phase II trials have evaluated the activity and efficacy of the potent mTOR inhibitor ridaforolimus, in combination with the anti-IGF1R dalotuzumab in patients with luminal MBC progressing after NSAIs [16, 17]. Both trials were negative, showing neither survival improvement nor differences in response rate. Furthermore, the association of dalotuzumab with ridaforolimus was always associated with high rates of adverse events $[16,17]$. 
Importantly, mTOR inhibitors have also been tested in luminal MBC in combination with chemo- and targeted therapies. Yardley and colleagues [18] recently published the results of a randomized phase II trial in which patients were randomized to receive paclitaxel and bevacizumab with or without everolimus as first-line treatment for HER2- MBC. The vast majority of patients enrolled had luminal BC (79\% for both arms). Again in this case, no significant differences in response rate $(p=0.49)$, PFS $(p=0.8859)$ and OS ( $p=0.4572)$ were observed. Additional randomized trials testing everolimus in luminal MBC are still ongoing. The DETECT IV is a single-arm phase II trial with the primary objective of estimating the efficacy of everolimus in combination with ET in postmenopausal patients with luminal BC and exclusively HER2-negative circulating tumor cells (CTCs) [19]. This study also aims at providing a better understanding of the prognostic and predictive value of CTCs in this clinical setting and evaluating the molecular features of CTCs [19]. Another randomized study is investigating the addition of everolimus to vinorelbine as second-line therapy for advanced HER2- MBC [20]. Finally, the BOLERO 6 phase II trial is assessing the efficacy of everolimus + exemestane compared to everolimus or capecitabine monotherapy in luminal HER2-negative MBC. The study is still ongoing, although patient recruitment is completed [21].

Besides all the aforementioned studies conducted in the metastatic setting, the adjuvant randomized phase III SWOG S1207 and UNICANCER trials are also ongoing. Both studies will explore the efficacy of everolimus associated with adjuvant ET in patients with high-risk non-metastatic, luminal BC [22, 23].

The story of PI3K inhibitors in luminal BC is quite controversial, as clinical trial results have been partly disappointing and have highlighted safety concerns. The PI3K inhibitors initially tested in phase II and III trials are defined as pan-PI3K inhibitors, since they act on all PI3K catalytic subunits.

Krop et al. [24] assessed the efficacy of the pan-PI3K inhibitor pictilisib in luminal AI-resistant MBC in the phase II randomized FERGI trial. This study was divided into 2 parts, with part 1 including patients with or without PIK3CA mutations, and part 2 including only patients with PIK3CA mutations. Patients were randomized to receive either fulvestrant + pictilisib or fulvestrant + placebo. No significant survival improvement was associated with the addition of pictilisib to fulvestrant in either part 1 or part 2. Furthermore, in part 1 , the experimental arm was more toxic than the control arm with grade 3 or worse adverse events occurring in $61 \%$ compared with $28 \%$ of patients, respectively. Of note, the proportion of patients discontinuing the experimental treatment due to toxicity was quite high (18\% in part 1 and $24 \%$ in part 2) and possibly contributed to the study failure [24]. More recently, the BELLE-2 trial evaluated another pan-PI3K inhibitor, named buparlisib, in association with fulvestrant in 1,147 patients with luminal HER2- MBC pretreated with AI. In the overall population, median PFS was significantly improved by the addition of buparlisib to ET (6.9 vs. 5.0 months; hazard ratio 0.78 , $\mathrm{p}=0.00021$ ) [25]. Importantly, the benefit associated with buparlisib was limited to patients with PIK3CA mutations detectable in their circulating tumor DNA (ctDNA) [25]. The most common grade 3-4 adverse events in the buparlisib group versus the placebo group were hepatotoxicity with increased ALT (25\% vs. $1 \%$ ) and AST $(18 \%$ vs. $3 \%)$, hyperglycemia $(15 \%$ vs. $<1 \%)$ and rash ( $8 \%$ vs. none). Moreover, patients in the experimental arm were frequently affected by depression (all grades $27 \%$ vs. $9 \%$ ) and anxiety (all grades $22 \%$ vs. $8 \%$ ) [23]. Positive results were also observed with the combination of fulvestrant + buparlisib in a more difficult therapeutic scenario, in the phase III BELLE-3 trial, which enrolled 432 patients who had progressed on or after therapy with everolimus. Previous treatment with 1 or more lines of chemotherapy was also allowed [26]. This trial demonstrated a significant improvement in PFS for the experimental arm (3.9 vs. 1.8 months; hazard ratio $0.67, \mathrm{p}<0.001$ ). Remarkably, subgroup analyses showed selective efficacy for the combination in PIK3CA-mutant patients, based on the analysis of both primary tumor specimens $(\mathrm{p}<0.001)$ and ctDNA $(\mathrm{p}<0.001)$ [26]. The most common grade 3-4 adverse events in the buparlisib group versus the placebo group were again hepatotoxicity and hyperglycemia. Mood disorders (depression and anxiety) were also frequent [26]. Recently, preclinical evidence suggested that selective inhibition of the $\mathrm{p} 110 \mathrm{a}$ isoform of PI3K could provide higher tolerability without affecting treatment efficacy [27-30]. The randomized phase III SOLAR-1 [31] and SANDIPER [32] trials are currently testing the new potent and selective PI3Ka inhibitors alpelisib and taselisib, respectively, in postmenopausal patients with $\mathrm{HR}^{+} / \mathrm{HER} 2^{-} \mathrm{MBC}$ progressing on or after an AI. Results from these trials are eagerly awaited to determine whether PI3K inhibition can be considered as a valid therapeutic option for luminal BC.

\section{Clinical Use of mTOR and PI3K Inhibitors in Luminal MBC: Current Indications and Future Directions}

For $\mathrm{HR}^{+} / \mathrm{HER} 2^{-} \mathrm{MBC}$ patients, ET represents the mainstay of treatment in the absence of visceral symptomatic disease. Therefore, patients with luminal de novo or recurrent MBC should be treated with as many endocrine-based therapies as possible to achieve an optimal efficacy/toxicity balance $[33,34]$. The recent introduction in clinical practice of new agents able to inhibit key mechanisms of endocrine resistance supports this strategy and allows the administration of chemotherapy to be delayed, radically changing the therapeutic scenario of luminal MBC.

As described above, solid clinical evidence supports the use of mTOR and PI3K inhibitors in luminal MBC. However, to date, everolimus is the only $\mathrm{PI} 3 \mathrm{~K} / \mathrm{AKT} / \mathrm{mTOR}$ pathway inhibitor successfully introduced in clinical practice, in patients progressing on or after an NSAI. Despite the positive results of BELLE-2 and 3 studies, pan-PI3K inhibitors have not yet been approved for clinical use, probably due to their unfavorable safety profile. In this context, the ongoing SOLAR-1 and SANDIPER trials will clarify whether selective PI3Ka inhibitors could become new treatment options to be integrated in the therapeutic algorithm for luminal 
MBC. Moreover, direct inhibition of AKT has also been shown in preclinical studies and in early-stage clinical trials to be a potential alternative therapeutic option to mTOR inhibitors [35]. Several phase II trials are underway addressing this open question [35].

Importantly, over the last 2 years, practice-changing clinical trials [36-40] have initiated a therapeutic revolution in luminal BC, demonstrating the utility of inhibiting another key mechanism of endocrine resistance, relying on the regulation of cell cycle, via the cyclin/CDK/Rb pathway. The randomized PALOMA-2 and MONALEESA-2 trials have led to the introduction of the CDK 4/6 inhibitors palbociclib and ribociclib, respectively, into clinical practice as first-line treatments in association with letrozole [37, 39]. Moreover, palbociclib has also been approved as a second-line therapy associated with fulvestrant, following the positive results of the PALOMA-3 trial [38]. These results have greatly improved the therapeutic possibilities for luminal $\mathrm{MBC}$, although defining the ideal treatment algorithm in this clinical setting remains a major challenge.

Due to the absence of trials directly comparing everolimus/ exemestane versus fulvestrant/palbociclib as second-line therapy, to date it is difficult to select patients who can benefit the most from either combination, and to define an ideal treatment sequence. In this scenario, a crucial research goal is to identify predictive biomarkers for the response to the new agents to develop personalized treatment strategies. Unfortunately, until now, no predictive biomarkers of the response to mTOR and CDK 4/6 inhibitors have been found [38, 41, 42]. Interestingly, data from case reports and from the SAFIR-01 trial suggest higher response to everolimus in cancer patients carrying rare MTOR [43], TSC1 and TSC2 [44], and AKT1 [45] mutations. These findings should be validated in larger prospective clinical trials. The scenario is different for PI3K inhibitors, as PIK3CA mutations, identified at the level of ctDNA, were predictive of response to buparlisib in both the BELLE- 2 and BELLE-3 studies $[25,26]$. Considering the non- invasive nature of the liquid biopsy approach, the findings of BELLE studies could represent a milestone in the development of personalized cancer therapies. In addition to defining the ideal sequential therapy with CDK $4 / 6$ and mTOR/PI3K inhibitors, another strategy to improve patient outcome might be the use of a combination of these agents. Recent preclinical studies suggested the potential utility of a 'triple association' of ET with both CDK $4 / 6$ and mTOR or PI3K inhibitors [46]. Therefore, several early-stage clinical trials are currently exploring this new potential strategy [47].

In conclusion, practice-changing clinical trials have successfully tested the addition of $\mathrm{mTOR} / \mathrm{PI} 3 \mathrm{~K}$ inhibitors to $\mathrm{ET}$ in luminal BC. In the absence of either clinical data or biomarkers to define the best treatment sequence, both the combinations of everolimus/ exemestane and fulvestrant/palbociclib represent the standard of care as second-line treatments in AI-resistant patients. The association of fulvestrant + pan-PI3K or selective PI3Ka inhibitors, especially in PI3KCA-mutant patients, as well as triple associations containing CDK 4/6 inhibitors, could be additional options in the near future. The use of liquid biopsy and its full implementation in the new clinical trials could be crucial for deciding 'which therapy to whom'.

\section{Acknowledgements}

Supported in part by the following Italian Grant Program: Programma Operativo Nazionale (PON) 2009-2013 01_01602. Ricerca e Competitività 2007-2013.

\section{Disclosure Statement}

None of the authors has conflicts of interests to disclose.

\section{References}

1 Sorlie T, Perou CM, Tibshirani R, et al.: Gene expression patterns of breast carcinomas distinguish tumor subclasses with clinical implications. Proc Natl Acad Sci USA 2001;98:10869-10874.

2 Schettini F, Buono G, Cardalesi C, et al.: Hormone receptor/human epidermal growth factor receptor 2-positive breast cancer: Where we are now and where we are going. Cancer Treat Rev 2016;46:20-26.

3 Osborne CK, Schiff R., Mechanisms of endocrine resistance in breast cancer. Annu Rev Med 2011;62:233-247.

4 Katso R, Okkenhaug K, Ahmadi K, et al.: Cellular function of phosphoinositide 3-kinases: Implications for development, immunity, homeostasis, and cancer. Annu Rev Cell Dev Biol 2001;17:615-675.

5 Yip CK, Murata K, Walz T, et al.: Structure of the human mTOR complex I and Its implications for rapamycin inhibition. Mol Cell 2010;38:768-774

6 Dey N, De P, Leyland-Jones B, Mechanisms and therapeutic advances in the management of endocrineresistant breast cancer. PI3K-AKT-mTOR inhibitors in breast cancers: From tumor cell signaling to clinical trials. Pharmacol Ther 2017;175:91-106.
7 Laplante M, Sabatini DM: mTOR signaling in growth control and disease. Cell 2012;149:274-293.

8 Baselga J, Campone M, Piccart M, et al.: Everolimus in postmenopausal hormone-receptor-positive advanced breast cancer. N Engl J Med 2012;366:520-529.

9 Piccart M, Hortobagyi GN, Campone M, et al.: Everolimus plus exemestane for hormone-receptorpositive, human epidermal growth factor receptor2-negative advanced breast cancer: Overall survival results from BOLERO-2. Ann Oncol 2014;25:2357-2362.

10 Bachelot T, Bourgier C, Cropet C, et al.: Randomized phase II trial of everolimus in combination with tamoxifen in patients with hormone receptor-positive, human epidermal growth factor receptor 2-negative metastatic breast cancer with prior exposure to aromatase inhibitors: A GINECO study. J Clin Oncol 2012;30:2718-2724.

11 Wolff AC, Lazar AA, Bondarenko I, et al.: Randomized phase III placebo-controlled trial of letrozole plus oral temsirolimus as first-line endocrine therapy in postmenopausal women with locally advanced or metastatic breast cancer. J Clin Oncol 2013;31:195-202.
12 Kornblum NS, Manola J, Klein P, et al.: PrECOG 0102:A randomized, double-blind, phase II trial of fulvestrant plus everolimus or placebo in post-menopausal women with hormone receptor (HR)-positive, HER2-negative metastatic breast cancer (MBC) resistant to aromatase inhibitor (AI) therapy. San Antonio Breast Cancer Symposium 2016, abstract S1-02.

13 Cardoso F, Villanueva C, Royce M, et al.: Everolimus (EVE) plus endocrine therapy in patients with estrogen receptor-positive $(\mathrm{ER}+)$, human epidermal growth factor receptor 2-negative (HER2-) advanced breast cancer (BC): First- and second-line data from the BOLERO-4 study. ASCO annual meeting 2017, abstract 1010.

14 Sun SY, Rosenberg LM, Wang X, et al.: Activation of Akt and eIF4E survival pathways by rapamycin-mediated mammalian target of rapamycin inhibition. Cancer Res 2005;65:7052-7058.

15 O'Reilly KE, Rojo F, She QB, et al.: mTOR inhibition induces upstream receptor tyrosine kinase signaling and activates Akt. Cancer Res 2006;66:1500-1508. 
16 Rugo HS, Trédan O, Ro J, et al.: A randomized phase II trial of ridaforolimus, dalotuzumab, and exemestane compared with ridaforolimus and exemestane in patients with advanced breast cancer. Breast Cancer Res Treat 2017; doi 10.1007/s10549-017-4375-5 [Epub ahead of print].

17 Baselga J, Morales SM, Awada A, et al.: A phase II study of combined ridaforolimus and dalotuzumab compared with exemestane in patients with estrogen receptor-positive breast cancer. Breast Cancer Res Treat 2017;163:535-544.

18 Yardley DA, Bosserman LD, O'Shaughnessy JA, et al.: Paclitaxel, bevacizumab, and everolimus/placebo as first-line treatment for patients with metastatic HER2negative breast cancer: A randomized placebo-controlled phase II trial of the Sarah Cannon Research Institute. Breast Cancer Res Treat 2015;154:89-97.

19 DETECT IV - a study in patients with HER2-negative metastatic breast cancer and persisting HER2-negative circulating tumor cells (CTCs). Available at: https:// clinicaltrials.gov [NLM Identifier: NCT02035813].

20 AIO-Studien-gGmbH: Study to compare vinorelbine in combination with the MTOR inhibitor everolimus vs vinorelbin monotherapy for second-line treatment in advanced breast cancer (VicTORia). Available at: https:// clinicaltrials. gov [NLM Identifier: NCT01520103].

21 A phase II study of everolimus in combination with exemestane versus everolimus alone versus capecitabine in advance breast cancer. (BOLERO-6). Available at: https://clinicaltrials.gov [NLM Identifier: NCT01783444].

22 Southwest Oncology Group. S1207 hormone therapy with or without everolimus in treating patients with breast cancer (e3). Available at: https://clinicaltrials. gov [NLM Identifier: NCT01674140].

23 Safety study of adding everolimus to adjuvant hormone therapy in women with high risk of relapse, ER+ and HER2- primary breast cancer, free of disease after receiving at least one year of adjuvant hormone therapy. Available at: https://clinicaltrials.gov [NLM Identifier: NCT01805271]

24 Krop IE, Mayer IA, Ganju V, et al.: Pictilisib for oestrogen receptor-positive, aromatase inhibitor-resistant, advanced or metastatic breast cancer (FERGI): A randomised, double-blind, placebo-controlled, phase 2 trial. Lancet Oncol 2016;17:811-821.

25 Baselga J, Im SA, Iwata $\mathrm{H}$, et al.: Buparlisib plus fulvestrant versus placebo plus fulvestrant in postmenopausal, hormone receptor-positive, HER2-negative, advanced breast cancer (BELLE-2): A randomised, double-blind, placebo-controlled, phase 3 trial. Lancet Oncol 2017;18:904-916.
26 Di Leo A, Seok Lee K, Ciruelos E, et al.: BELLE-3: A phase III study of buparlisib and fulvestrant in postmenopausal women with HR+, HER2-, aromatase inhibitor-treated, locally advanced or metastatic breast cancer, who progressed on or after mTOR inhibitorbased treatment. San Antonio Breast Cancer Symposium 2016, abstract S4-07.

27 Juric D, Argiles G, Burris HA, et al.: Phase I study of BYL719, an alpha-specific PI3K inhibitor, in patients with PIK3CA mutant advanced solid tumors: Preliminary efficacy and safety in patients with PIK3CA mutant ER-positive (ER+) metastatic breast cancer (MBC). Cancer Res 2012;72:abstract P6-10-07.

28 Olivero AG, Heffron TP, Baumgardner M, et al.: Discovery of GDC-0032: A beta-sparing PI3K inhibitor active against PIK3CA mutant tumors. Cancer Res 2013;73:abstract DDT02-01.

29 Saura C, Sachdev J, Patel MR, et al.: Phlb study of the PI3K inhibitor taselisib (GDC-0032) in combination with letrozole in patients with hormone receptor-positive advanced breast cancer. Cancer Res 2015;75:abstract PD5-2.

30 Juric D, Krop I, Ramanathan RK, et al.: GDC-0032, a beta isoform-sparing PI3K inhibitor: Results of a firstin-human phase Ia dose escalation study. Cancer Res 2013;73:abstract LB-64.

31 Rugo HS, Andre F, Rubovszky G, et al.: A phase 3 study of alpelisib (ALP) plus fulvestrant (FUL) in men and postmenopausal women with hormone receptorpositive $(\mathrm{HR}+)$, human epidermal growth factor receptor 2-negative (HER2-) ABC progressing on or after aromatase inhibitor (AI) therapy: SOLAR-1. J Clin Oncol 2017;35 suppl; abstr TPS1111.

32 Baselga J, Cortés J, DeLaurentiis M, et al.: SANDPIPER: Phase III study of the PI3-kinase (PI3K) inhibitor taselisib (GDC-0032) plus fulvestrant in patients (pts) with estrogen receptor (ER)-positive, HER2-negative locally advanced or metastatic breast cancer (BC) enriched for pts with PIK3CA-mutant tumors. J Clin Oncol 2017;35 suppl; abstr TPS1119.

33 Cardoso F, Costa A, Senkus E, et al.: 3rd ESO-ESMO International Consensus Guidelines for Advanced Breast Cancer (ABC 3). Ann Oncol 2017;28:16-33.

34 NCCN Breast Cancer Guidelines 2017, available at https://www.nccn.org/professionals/physician_gls/f_ guidelines.asp\#site. Last access on 06/07/2017.

35 Bahrami A, Khazaei M, Shahidsales S, et al.: The therapeutic potential of PI3K/Akt/mTOR inhibitors in breast cancer: Rational and progress. J Cell Biochem 2017; doi: 10.1002/jcb.26136 [Epub ahead of print].
36 Finn RS, Crown JP, Ettl J, et al.: Efficacy and safety of palbociclib in combination with letrozole as first-line treatment of ER-positive, HER2-negative, advanced breast cancer: expanded analyses of subgroups from the randomized pivotal trial PALOMA-1/TRIO-18. Breast Cancer Res 2016;18:67.

37 Finn RS, Martin M, Rugo HS, et al.: Palbociclib and letrozole in advanced breast cancer. N Engl J Med 2016;375:1925-1936.

38 Cristofanilli M, Turner NC, Bondarenko I, et al.: Fulvestrant plus palbociclib versus fulvestrant plus placebo for treatment of hormone-receptor-positive, HER2-negative metastatic breast cancer that progressed on previous endocrine therapy (PALOMA-3): Final analysis of the multicentre, double-blind, phase 3 randomised controlled trial. Lancet Oncol 2016;17:425-439.

39 Hortobagyi G, Stemmer SM, Burris HA, et al.: Ribociclib as first-line therapy for HR-positive, advanced breast cancer. N Engl J Med 2016;375:1738-1748.

40 Sledge GW Jr, Toi M, Neven P, et al.: MONARCH 2: Abemaciclib in combination with fulvestrant in women with HR+/HER2- advanced breast cancer who had progressed while receiving endocrine therapy. J Clin Oncol 2017;35:2875-2884.

41 Hortobagyi GN, Chen D, Piccart M, et al.: Correlative analysis of genetic alterations and everolimus benefit in hormone receptor-positive, human epidermal growth factor receptor 2-negative advanced breast cancer: Results from BOLERO-2. J Clin Oncol 2015;34:419-426.

42 Fribbens C, O'Leary B, Kilburn L, et al.: Plasma ESR1 mutations and the treatment of estrogen receptor-positive advanced breast cancers. J Clin Oncol 2016;34: 2961-2968.

43 Wagle N, Grabiner BC, Van Allen EM, et al.: Activating mTOR mutations in a patient with an extraordinary response on a phase I trial of everolimus and pazopanib. Cancer Discov 2014;4:546-553.

44 Iyer G, Hanrahan AJ, Milowsky MI, et al.: Genome sequencing identifies a basis for everolimus sensitivity. Science 2012;338:221.

45 Andre F, Bachelot T, Commo F, et al.: Comparative genomic hybridisation array and DNA sequencing to direct treatment of metastatic breast cancer: A multicentre, prospective trial (SAFIR01/ UNICANCER). Lancet Oncol 2014;15:267-274.

46 Vora SR, Juric D, Kim N, et al.: CDK 4/6 inhibitors sensitize PIK3CA mutant breast cancer to PI3K inhibitors. Cancer Cell 2014;26:136-149.

47 Augereau P, Patsouris A, Bourbouloux E, et al.: Hormonoresistance in advanced breast cancer: A new revolution in endocrine therapy. Ther Adv Med Oncol 2017;9:335-346. 